\title{
Laser-based visible light communications and underwater wireless optical communications: a device perspective
}

Chao Shen, Omar Alkhazragi, Xiaobin Sun, Yujian Guo, Tien Khee $\mathrm{Ng}$, et al.

Chao Shen, Omar Alkhazragi, Xiaobin Sun, Yujian Guo, Tien Khee Ng, Boon S. Ooi, "Laser-based visible light communications and underwater wireless optical communications: a device perspective ," Proc. SPIE 10939, Novel In-Plane Semiconductor Lasers XVIII, 109390E (1 March 2019); doi: 10.1117/12.2504681

SPIE. Event: SPIE OPTO, 2019, San Francisco, California, United States 


\title{
Laser-based visible light communications and underwater wireless optical communications: a device perspective
}

\author{
(invited paper) \\ Chao Shen, Omar Alkhazragi, Xiaobin Sun, Yujian Guo, Tien Khee Ng, Boon S. Ooi* \\ Photonics Laboratory, King Abdullah University of Science and Technology (KAUST), 4700 \\ KAUST, Thuwal, Saudi Arabia 21534
}

\begin{abstract}
High-speed visible light communications (VLC) has been identified at an essential part of communication technology for $5 \mathrm{G}$ network. VLC offers the unique advantages of unregulated and secure channels, free of EM interference. Compared with the LED-based VLC transmitter, laser-based photonic systems are promising for compact, droop-free, and high-speed white lighting and VLC applications, ideal for ultra-fast 5G network and beyond. Besides the potential for achieving high data rate free-space communication links, i.e. the Li-Fi network, laser-based VLC technology can also enable underwater wireless optical communications (UWOC) for many important applications.

In this paper, the recent research progress and highlights in the fields of laser-based VLC and UWOC have been reviewed with a focused discussion on the performance of various light sources, including the modulation characteristics of GaNbased edge emitting laser diodes (EELDs), superluminescent diodes (SLDs) and vertical-cavity surface-emitting lasers (VCSELs). Apart from the utilization of discrete components for building transceiver in VLC systems, the development of III-nitride laser-based photonic integration has been featured. Such on-chip integration offers many advantages, including having a small-footprint, high-speed, and low power consumption.

Finally, we discuss the considerations of wavelength selection for various VLC and UWOC applications. Comparison of infrared (IR) and visible lasers for channels with high turbulence and the study of ultraviolet (UV) and visible lasers for non-line-of-sight (NLOS) communications are presented.
\end{abstract}

Keywords: Gallium Nitride, laser diode, semiconductor laser, visible light communication, Li-Fi, solid state lighting, optical wireless communication, underwater communication.

\section{OVERVIEW OF LASER BASED VLC AND UWOC RESEARCH}

The use of light sources such as laser diodes (LD) and light emitting diodes (LED) emitting in the visible band of the electromagnetic spectrum as means of data transmission offers numerous advantages. For example, these sources can provide high data rates while simultaneously serving as efficient lighting sources. They can also transmit the data at long distance in both free space channel and water channel, making it a promising technology for enabling Internet of underwater things (IoUT). In this section, we will present an overview of the research achievements in laser-based visible light communications (VLC) and underwater wireless optical communications (UWOC).

\subsection{Laser-based VLC}

Laser-based VLC has seen remarkable advancements in recent years, which are summarized in Figure 1 [1-19] where the data rates are plotted versus the distance of transmission. The use of LDs in VLC has many advantages over LEDs such as having a much higher modulation bandwidth and enabling narrow beam transmission for long distance applications. For instance, a 100-m VLC link was demonstrated with a data rate of $2.3 \mathrm{Gbps}$ was achieved using on-off keying (OOK) with a bit error ratio (BER) of $1.8 \times 10^{-3}$, which is below the forward error correction (FEC) limit of $3.8 \times 10^{-3}$ [4].

*boon.ooi@kaust.edu.sa; phone 96612 808-4350; photonics.kaust.edu.sa

Novel In-Plane Semiconductor Lasers XVIII, edited by Alexey A. Belyanin,

Peter M. Smowton, Proc. of SPIE Vol. 10939, 109390E · C 2019 SPIE

CCC code: $0277-786 \mathrm{X} / 19 / \$ 18 \cdot$ doi: $10.1117 / 12.2504681$

Proc. of SPIE Vol. 10939 109390E-1 
However, there is a trade-off between the transmission distance and the quality of the white lighting to be taken into consideration. Depending on the application, a collimated beam generated by laser diodes would be preferred for longdistance transmission, but a divergent beam can be preferred for lighting. While the OOK modulation technique is simple and thus, commonly used [2, 4-6], there are many other modulation schemes that have been used in laser-based VLC. The use of more spectral efficient techniques, such as orthogonal frequency division multiplexing (OFDM), has shown great results in increasing the data rates of VLC systems [7, 13, 15]. Other methods, including pulse amplitude modulation (PAM) [18] and carrier-less amplitude and phase (CAP) modulation [1], are also useful techniques that have been demonstrated.

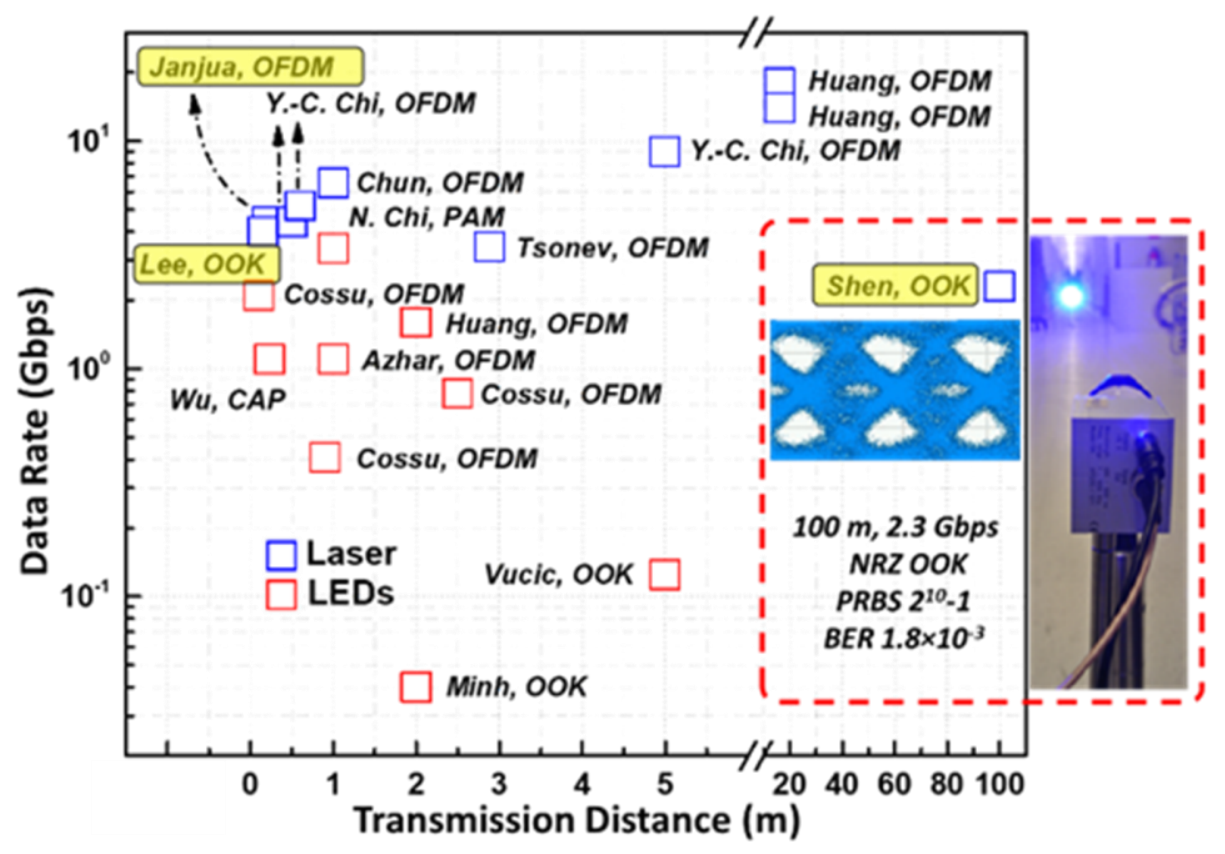

Figure 1. A summary of research progress in laser-based VLC and its comparison with LED-based VLC, showing the data rates and the transmission distances. (Data set can be accessed at https://photonics.kaust.edu.sa/Pages/Research-VLC.aspx)

\subsection{Laser-based UWOC}

Laser-based UWOC has attracted many researchers due to the variety of applications in which it can be deployed. These applications include underwater pipeline monitoring and maintenance, oceanography and environmental studies, and military applications. Laser-based UWOC become attractive due to the fact that the conventional acoustic communications cannot meet the bandwidth demands in many applications, such as live video streaming [20-23], and they also suffer from high latency. While radio frequency (RF) can be used for underwater wireless communication, it suffers from very high attenuation in water which limits the transmission range [24-26]. Because of the limitations of the aforementioned alternatives, UWOC has received a lot of attention in recent years, and Figure 2 shows the recent progress in the field [2743]. These demonstrations range from test-bed experiments, such as presented in [32], and real environment tests [42]. The modulation schemes and multiplexing techniques used in VLC can also be implemented in UWOC. Furthermore, in UWOC, non-line-of-sight (NLOS) communication has been demonstrated [44], which removes the strict alignment conditions between the transmitter and the receiver. In a NLOS system, the scattering of the light in water is utilized since it can help in directing the randomly-scattered photons toward the receiver, which will be discussed in a later section. A comprehensive review related to the transmitter and receiver technologies, key components, underwater optical channel models, and effects of turbulence in UWOC systems has recently published [45]. 


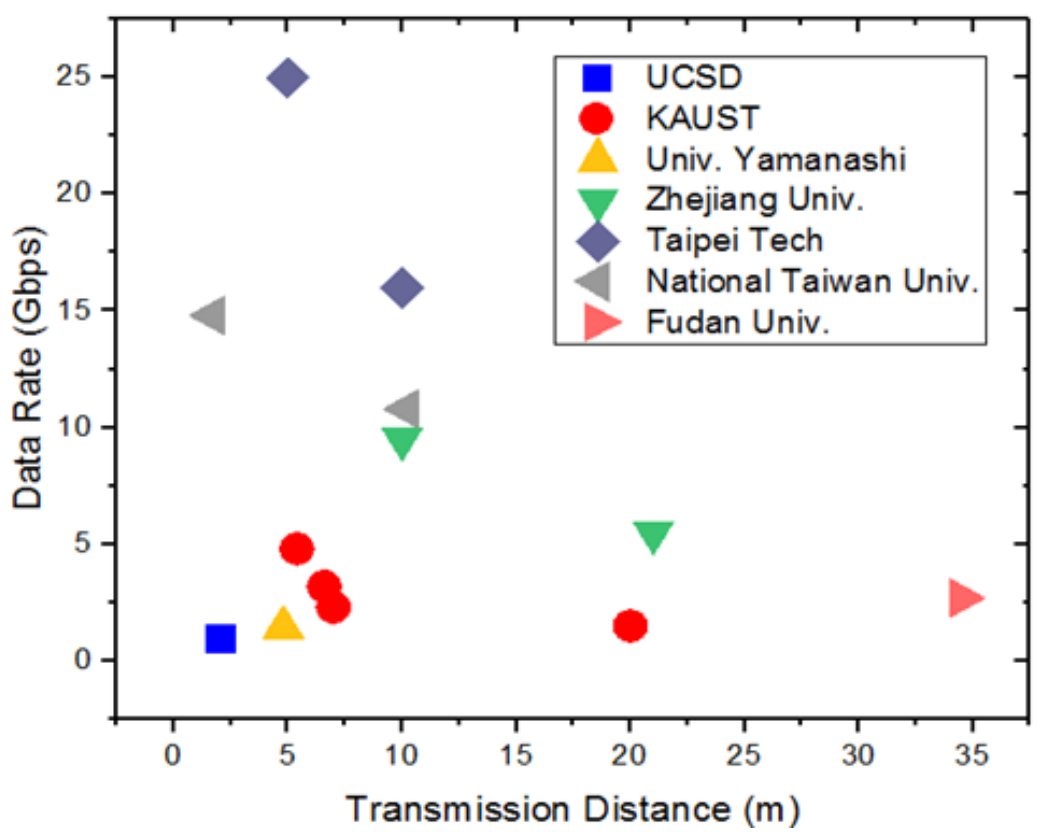

Figure 2. A summary of research progress in laser-based UWOC, showing the data rates and the transmission distances. (Data set can be accessed at https://photonics.kaust.edu.sa/Pages/Underwater-Wireless-Optical-Communication.aspx)

\section{GAN BASED EELD, SLD AND VCSEL}

In laser-based VLC and UWOC systems, the light source is one of the key components to enable high-speed data communication. Since the blue-green light emitters were based on GaN materials, the high-speed VLC system requires a fast GaN-based transmitter. There are three kinds of transmitters including the edge emitting laser diodes (EELDs), superluminescent diodes (SLDs) and vertical-cavity surface-emitting lasers (VCSELs).

The first demonstration of pulsed-operated InGaN/GaN/AlGaN-based room-temperature EELD was reported by Nakamura et al. [46]. Since then, the reliability of InGaN-based lasers has improved significantly, and the output power has been increased from a few $\mathrm{mW}$ to a few $\mathrm{W}$ per diode [47]. A high power GaN laser diode with output power over $7 \mathrm{~W}$ was reported in [48]. The modulation characteristics of GaN-based laser diodes were studied in various works for VLC and UWOC applications [49-55]. A $1 \mathrm{GHz}$ modulation bandwidth was measured in a commercial high power blue laser diode [55] and a later study suggested the modulation bandwidth for GaN-based violet laser could reach beyond $5 \mathrm{GHz}$ [56]. Utilizing the injection locking technique, it has been reported that the bandwidth of the laser diode can be enhanced, making it promising for high data rate VLC systems [50].

The GaN-based SLDs are light emitters generating amplified spontaneous emission (ASE). SLDs have been found to have attractively-combined optical characteristics, i.e. a broadband spectrum similar to that of light-emitting diodes (LEDs) and high spatial coherence similar to that of LDs $[57,58]$. SLDs are typically used as the source in optical coherence tomography (OCT) and fiber optic gyroscopes (FOG) systems. Recently, high power and high speed violet-blue SLDs have been reported, suggesting its potential for VLC and UWOC applications [59]. In one of the earliest demonstrations, a blue SLD emitting at $420 \mathrm{~nm}$ was demonstrated to have an output power of $100 \mathrm{~mW}$ in pulse mode and $2.8 \mathrm{~mW}$ in continuous wave (CW) operation [13]. Since then, there are continuous reports of III-nitride SLDs with increasing optical power, such as over $100 \mathrm{~mW}$ blue SLD in CW operation [60], $350 \mathrm{~mW}$ violet SLD in CW operation [35], and $474 \mathrm{~mW}$ blue SLD in pulse operation [61]. The modulation characteristics in GaN-based SLD is first studied by Shen et al. showing a modulation bandwidth of $560 \mathrm{MHz}$ [62]. Utilizing violet-blue SLDs as transmitters, the VLC links with data rates of 1.3 Gbps [63] and 1.45 Gbps [61] have been reported, suggesting their attractive properties for VLC applications.

VCSELs are ideal for high speed operation owing to their short cavity and small form factor [64]. However, unlike conventional VCSEL based on distributed Bragg reflectors (DBRs) based on lattice matching material system, such as GaAs/AlGaAs, GaN based VCSEL suffers from the large lattice-mismatch AlGaN/GaN DBRs, leading to cracking of 
material structure after growth. Until very recently, the output power of the violet-blue VCSEL increased from $\mu \mathrm{W}$ to above $10 \mathrm{~mW}$ [65]. Therefore, significant efforts are required to develop high power, high speed GaN-based VCSELs. The recent advances and challenges of GaN-based VCSELs are summarized in [66]. Nonetheless, the investigation of nonpolar InGaN/GaN quantum-well VCSEL shows a modulation bandwidth beyond $\mathrm{GHz}$ [67], confirming the fact that GaN-based VCSEL has great potential for high-speed VLC transmitters.

\section{INTEGRATED PHOTONICS AT VISIBLE COLOR REGIME}

GaAs- and InP-based photonic integration has been achieved in telecommunication wavelength regime for many commercial applications [68]. Though there are significant advances in III/V photonic integrated circuits (PIC) in far-red and infrared wavelength regime, the realization of photonics integration in the visible wavelength regime remains a challenging topic. There are a few studies on integrating different optical functionalities with GaN-based LEDs, photodetectors in particular. Monolithic integration of nitride multi-quantum well (MQW) LEDs with ultraviolet (UV) Schottky barrier PDs has been demonstrated by Jiang et al. [69]. Such devices can function as the transmitters to emit visible light signals, and as receivers to analyze incoming UV signals for bi-directional optical wireless communication applications. By joining a forward biased LED, a suspended waveguide, and a reverse biased PD together, an integrated p-i-n junction device sharing the same InGaN/GaN MQW active region has been demonstrated on GaN-on-silicon platform [70]. Apart from the planar structures, Tchernycheva et al. reported a nanowire photonic platform consisting of InGaN core-shell nanowire LEDs and PDs optically coupled by SiN waveguides [71].

Recently, we have discussed and demonstrated the first monolithic integration of EELDs together with modulators, amplifiers, and detectors, for VLC applications [72]. The first blue-emitting integrated waveguide modulator-laser diode with a high modulation efficiency of $2.68 \mathrm{~dB} / \mathrm{V}$ has been demonstrated in [73]. For higher efficiency, group-III-nitride electro-absorption modulator operating at a reduced modulation bias, semipolar (20 $\overline{21}$ ) MQWs was utilized and characterized [73-75]. The modulation effect, as evident from the red-shifting of absorption edge, is due to external-fieldinduced quantum-confined-Stark-effect (QCSE) [73]. An integrated short-wavelength semiconductor optical amplifier with the laser diode at $\sim 404 \mathrm{~nm}$ is demonstrated with a large gain of $5.32 \mathrm{~dB}$ at $6 \mathrm{~V}$ [74]. The integrated waveguide modulator shows a modulation bandwidth of $1 \mathrm{GHz}$, which enables Gbps data rate VLC links. The integrated GaN-based semiconductor optical amplifier (SOA) - laser diode was demonstrated in [76], showing a large gain of $5.7 \mathrm{~dB}$. By using the modulated amplifier scheme, the SOA-LD shows a high-speed modulation capability of Gbps [77]. Hence, this device platform will also be useful as the transmitter in VLC systems. Besides, a high-performance InGaN-based waveguide photodetector integrated LD sharing the single active region is presented [78] with a responsivity of $0.051 \mathrm{~A} / \mathrm{W}$ at $405 \mathrm{~nm}$ and a large modulation bandwidth of $230 \mathrm{MHz}$. The results are significant in developing the platform technology to enable III-nitride PIC for smart lighting and display, VLC, optical switching, clocking and optical interconnect [79].

\section{WHEN TO CONSIDER LONG WAVELENGTH LASERS}

The visible-light-based VLC and UWOC links were associated with attenuation and turbulence effects, and those effects are in particular important for UWOC. The working distance of the communication system is largely depending on the power attenuation caused by the absorption and scattering effects during the propagation of optical signals.

The performance of UWOC links is severely limited by optical turbulence in the water channel which is mainly due to temperature and salinity fluctuations. Optical turbulence induces random temporal and spatial irradiance and phase fluctuations (scintillations) which ultimately deteriorate the optical quality and the coherence properties of laser beams leading to performance degradation $[80,81]$.

Detailed understanding of scintillations in underwater turbulent media is crucial in evaluating the reliability of UWOC systems. The probability density functions of laser beam intensity fluctuations due to salty and bubbly underwater channels under various channel conditions were experimentally obtained by Jamali [82]. A more comprehensive study to characterize turbulence-induced fading in UWOC channels in the presence of air bubbles for fresh and salty waters, based on experimental data was proposed by Zedini et al. [83]. Recently, radiations with long wavelength (red light) are reported to outperform shorter wavelengths in terms of circumventing the effect of turbulence [84].

The scattering effect, which is also a wavelength-dependent phenomenon, results in the changes of direction of the beam propagation. Theoretical- and simulation-based studies have been carried out to investigate the inherent characteristics of 
the UWOC channel and examine the feasibility of the related mitigation strategies $[85,86]$. The feasibility of using Maalox to emulated the scattering scenario in the real seawater was validated by Laux et al [87]. Lee et al. reported on the experimental investigation of the effects of particulates in-suspension with increasing concentrations in the water medium on the optical beam for underwater wireless transmission [88, 89]. The Gbps NIR-based UWOC system is also feasible. UWOC systems with long wavelength light sources are more resilient to the effects of particulates in-suspension over the underwater channel with a smaller scattering effect, as evident by the smaller variations in transmittance. The forward scattering potentially directs the resultant beam towards the receiver. The adoption of directly modulated long wavelength LDs would spur the realization of future high-speed, large-bandwidth and long-distance UWOC systems that are robust to different water types.

\section{WHEN TO CONSIDER SHORT WAVELENGTH LASERS}

While most of the high-speed VLC and UWOC links were based on line-of-sight (LOS) configurations, there are strict requirements on positioning, acquisition, and tracking (PAT). However, turbulence [90, 91], turbidity [92], and obstacles [90] in the air or water channel are reported to cause severe signal fading, and even complete signal loss, in LOS VLC/UWOC links. To this end, non-line-of-sight (NLOS) communication links can be implemented to mitigate the abovementioned issues. Such studies are important for laser-based UWOC systems to address issues related to the PAT requirements. Diffuse-LOS and NLOS UWOC links can be established either through light reflection from the water surface [93] or light scattering [94] from the molecules in the water. Therefore, short-wavelength, including UV light emitters are typically favorable to be utilized in NLOS UWOC owning to the high scattering coefficients in this range of wavelength.

There are Monte Carlo simulations [95] and the Henyey-Greenstein (HG) phase function [96] studies to develop models describing the transmitted photons' trajectory. A recent demonstration of NLOS UWOC link reveals the advantage of using a short wavelength, 375-nm laser [12] to achieve enhanced NLOS UWOC link and the path loss has been reported under various geometries. The experiments suggest that path loss decreases with smaller azimuth angles, higher water turbidity, and shorter wavelengths due in part to enhanced scattering utilizing short wavelength lasers.

\section{CONCLUSIONS}

In this paper, the recent progress in laser-based visible light communications and underwater wireless optical communications was discussed with a focus on light emitting devices. The advantages, properties, optical power, and modulation characteristics of GaN-based edge emitting laser diodes, superluminescent diodes and vertical-cavity surfaceemitting lasers have been introduced. The pathway towards integrated photonics in the visible color regime using IIInitride laser platform for high-speed applications is reviewed. The work also features the utilization of long wavelength lasers for VLC and UWOC links in high turbulence channels and the utilization of short wavelength lasers for non-lineof-sight VLC and UWOC links.

\section{ACKNOWLEDGEMENT}

The authors acknowledge the financial support from King Abdulaziz City for Science and Technology (KACST) Grant No. KACST TIC R2-FP-008, and KACST-KAUST-UCSB Solid-State Lighting Program. This publication is based on work supported by the King Abdullah University of Science and Technology (KAUST) (baseline funding, BAS/1/161401-01, KAUST funding KCR/1/2081-01-01, and GEN/1/6607-01-01).

\section{REFERENCES}

[1] F. Wu, C. Lin, C. Wei et al., "1.1-Gb/s White-LED-Based Visible Light Communication Employing Carrier-Less Amplitude and Phase Modulation," IEEE Photon. Technol. Lett., 24(19), 1730-1732 (2012).

[2] J. Vucic, C. Kottke, S. Nerreter et al., "125 Mbit/s over $5 \mathrm{~m}$ wireless distance by use of OOK-Modulated phosphorescent white LEDs." 1-2.

[3] D. Tsonev, S. Videv, and H. Haas, "Towards a $100 \mathrm{~Gb} / \mathrm{s}$ visible light wireless access network," Opt. Express, 23(2), 1627-1637 (2015). 
[4] B. Ooi, "Visible Light Communication.", Pacific Rim Conference on Lasers and Electro-Optics (CLEO-PR) (2018).

[5] H. L. Minh, D. O. Brien, G. Faulkner et al., "High-Speed Visible Light Communications Using Multiple-Resonant Equalization," IEEE Photon. Technol. Lett., 20(14), 1243-1245 (2008).

[6] C. Lee, C. Zhang, M. Cantore et al., "4 Gbps direct modulation of $450 \mathrm{~nm}$ GaN laser for high-speed visible light communication,” Opt. Express, 23(12), 16232-16237 (2015).

[7] B. Janjua, H. M. Oubei, J. R. D. Retamal et al., "Going beyond 4 Gbps data rate by employing RGB laser diodes for visible light communication," Opt. Express, 23(14), 18746-18753 (2015).

[8] Y.-F. Huang, C.-T. Tsai, H.-Y. Kao et al., "17.6-Gbps Universal Filtered Multi-Carrier Encoding of GaN Blue LD for Visible Light Communication," OSA Technical Digest. STh1C.5 (2017).

[9] Y.-F. Huang, Y.-C. Chi, H.-Y. Kao et al., "Blue Laser Diode Based Free-space Optical Data Transmission elevated to 18 Gbps over 16 m," Sci. Rep., 7(1), 10478 (2017).

[10] X. Huang, Z. Wang, J. Shi et al., "1.6 Gbit/s phosphorescent white LED based VLC transmission using a cascaded pre-equalization circuit and a differential outputs PIN receiver," Opt. Express, 23(17), 22034-22042 (2015).

[11]G. Cossu, M. Presi, R. Corsini et al., "A Visible Light localization aided Optical Wireless system." 802-807.

[12] G. Cossu, A. M. Khalid, P. Choudhury et al., "Long distance indoor high speed visible light communication system based on RGB LEDs." 2012 Asia Communications and Photonics Conference (ACP), 1-3. (2012)

[13] G. Cossu, A. M. Khalid, P. Choudhury et al., "2.1 Gbit/s Visible Optical Wireless Transmission," OSA Technical Digest. P4.16 (2012).

[14]H. Chun, S. Rajbhandari, D. Tsonev et al., "Visible light communication using laser diode based remote phosphor technique." 2015 IEEE International Conference on Communication Workshop (ICCW), 1392-1397 (2015).

[15]Y.-C. Chi, D.-H. Hsieh, C.-T. Tsai et al., "450-nm GaN laser diode enables high-speed visible light communication with 9-Gbps QAM-OFDM,” Opt. Express, 23(10), 13051-13059 (2015).

[16] Y.-C. Chi, D.-H. Hsieh, C.-Y. Lin et al., "Phosphorous Diffuser Diverged Blue Laser Diode for Indoor Lighting and Communication," Sci. Rep., 5, 18690 (2015).

[17] Y. Chi, Y. Huang, T. Wu et al., "Violet diode laser based 11.2-Gbit/s point-to-point and 4.4-Gbit/s white lighting communications." 2017 Conference on Lasers and Electro-Optics Europe \& European Quantum Electronics Conference (2017).

[18]N. Chi, M. Zhang, Y. Zhou et al., "3.375-Gb/s RGB-LED based WDM visible light communication system employing PAM-8 modulation with phase shifted Manchester coding," Opt. Express, 24(19), 21663-21673 (2016).

[19] A. H. Azhar, T. Tran, and D. O. Brien, "A Gigabit/s Indoor Wireless Transmission Using MIMO-OFDM VisibleLight Communications,” IEEE Photon. Technol. Lett., 25(2), 171-174 (2013).

[20]C. Xilin, Q. Fengzhong, and Y. Liuqing, "Single carrier FDMA over underwater acoustic channels." 1052-1057.

[21]S. Wei, L. Yurong, L. Wenhui et al., "Study of single-carrier coherent high-speed underwater acoustic communication." 2013 OCEANS - San Diego (2013).

[22]B. Li, S. Zhou, M. Stojanovic et al., "Multicarrier Communication Over Underwater Acoustic Channels With Nonuniform Doppler Shifts," IEEE J. Ocean. Eng., 33(2), 198-209 (2008).

[23]H. Kaushal, and G. Kaddoum, "Underwater Optical Wireless Communication," IEEE Access, 4, 1518-1547 (2016).

[24]J. Shi, S. Zhang, and C. Yang, "High frequency RF based non-contact underwater communication." 2012 Oceans - Yeosu (2012).

[25]J. Lloret, S. Sendra, M. Ardid et al., "Underwater Wireless Sensor Communications in the $2.4 \mathrm{GHz}$ ISM Frequency Band," Sensors, 12(4), (2012).

[26]X. Che, I. Wells, G. Dickers et al., "Re-evaluation of RF electromagnetic communication in underwater sensor networks," IEEE Commun. Mag., 48(12), 143-151 (2010).

[27] J. Xu, Y. Song, X. Yu et al., "Underwater wireless transmission of high-speed QAM-OFDM signals using a compact red-light laser," Opt. Express, 24(8), 8097-8109 (2016).

[28]J. Xu, M. Kong, A. Lin et al., "Directly modulated green-light diode-pumped solid-state laser for underwater wireless optical communication," Opt. Lett., 42(9), 1664-1667 (2017).

[29]T.-C. Wu, Y.-C. Chi, H.-Y. Wang et al., "Blue Laser Diode Enables Underwater Communication at 12.4 Gbps," Sci. Rep., 7, 40480-40480 (2017).

[30]P. Tian, X. Liu, S. Yi et al., "High-speed underwater optical wireless communication using a blue GaN-based micro-LED," Opt. Express, 25(2), 1193-1201 (2017). 
[31] J. B. Snow, J. P. Flatley, D. E. Freeman et al., "Underwater propagation of high-data-rate laser communications pulses." San Diego '92, 1750, 9 (1992).

[32]C. Shen, Y. Guo, H. M. Oubei et al., "20-meter underwater wireless optical communication link with $1.5 \mathrm{Gbps}$ data rate," Opt. Express, 24(22), 25502-25509 (2016).

[33]H. M. Oubei, C. Li, K.-H. Park et al., "2.3 Gbit/s underwater wireless optical communications using directly modulated $520 \mathrm{~nm}$ laser diode," Opt. Express, 23(16), 20743-20748 (2015).

[34]H. M. Oubei, J. R. Duran, B. Janjua et al., "4.8 Gbit/s 16-QAM-OFDM transmission based on compact 450-nm laser for underwater wireless optical communication," Opt. Express, 23(18), 23302-23309 (2015).

[35] H. M. Oubei, J. R. Durán, B. Janjua et al., "Wireless optical transmission of $450 \mathrm{~nm}, 3.2 \mathrm{Gbit} / \mathrm{s}$ 16-QAM-OFDM signals over 6.6 m underwater channel." 2016 Conference on Lasers and Electro-Optics (2016).

[36] K. Nakamura, I. Mizukoshi, and M. Hanawa, "Optical wireless transmission of $405 \mathrm{~nm}, 1.45 \mathrm{Gbit} / \mathrm{s}$ optical IM/DD-OFDM signals through a 4.8 m underwater channel,” Opt. Express, 23(2), 1558-1566 (2015).

[37] H. Lu, C. Li, H. Lin et al., "An 8 m/9.6 Gbps Underwater Wireless Optical Communication System,” IEEE Photonics J., 8(5), 1-7 (2016).

[38]X. Liu, S. Yi, R. Liu et al., "34.5 m Underwater optical wireless communication with $2.70 \mathrm{Gbps}$ data rate based on a green laser with NRZ-OOK modulation." 14th China International Forum on Solid State Lighting: International Forum on Wide Bandgap Semiconductors (2017).

[39] C.-Y. Li, H.-H. Lu, W.-S. Tsai et al., "16 Gb/s PAM4 UWOC system based on 488-nm LD with light injection and optoelectronic feedback techniques," Opt. Express, 25(10), 11598-11605 (2017).

[40] Y. Huang, C. Tsai, Y. Chi et al., "Filtered Multicarrier OFDM Encoding on Blue Laser Diode for 14.8-Gbps Seawater Transmission," J. Lightwave Technol., 36(9), 1739-1745 (2018).

[41]F. Hanson, and S. Radic, "High bandwidth underwater optical communication," Appl. Optics, 47(2), 277-283 (2008).

[42]S. A. Hamilton, C. E. DeVoe, A. S. Fletcher et al., "Undersea narrow-beam optical communications field demonstration." Proc. SPIE, 10186, 16 (2017).

[43] Y. Chen, M. Kong, T. Ali et al., "26 m/5.5 Gbps air-water optical wireless communication based on an OFDMmodulated 520-nm laser diode," Opt. Express, 25(13), 14760-14765 (2017).

[44]X. Sun, W. Cai, O. Alkhazragi et al., "375-nm ultraviolet-laser based non-line-of-sight underwater optical communication," Opt. Express, 26(10), 12870-12877 (2018).

[45]H. M. Oubei, C. Shen, A. Kammoun et al., "Light based underwater wireless communications," Jpn. J. Appl. Phys, 57(8), (2018).

[46] S. Nakamura, and S. F. Chichibu, [Introduction to Nitride Semiconductor Blue Lasers and Light Emitting Diodes] CRC Press, (2000).

[47] S. Lutgen, A. Avramescu, T. Lermer et al., "Progress of blue and green InGaN laser diodes," SPIE, 7616, (2010).

[48] M. Kawaguchi, S. Nozaki, K. Morimoto et al., "High-power GaN diode lasers and their applications," Proceedings of the 2017 IEEE High Power Diode Lasers and Systems Conference, $43-44$ (2017).

[49] B. Xue, Z. Liu, J. Yang et al., "Characteristics of III-nitride based laser diode employed for short range underwater wireless optical communications," Opt. Commun., 410, 525-530 (2018).

[50] M. H. M. Shamim, M. A. Shemis, C. Shen et al., "Investigation of Self-Injection Locked Visible Laser Diodes for High Bit-Rate Visible Light Communication," IEEE Photonics J., 10(4), (2018).

[51]C. M. Lee, C. Shen, C. Cozzan et al., "Semipolar GaN-based laser diodes for Gbit/s white lighting communication: devices to systems," Proc. SPIE, 10532 (2018).

[52]F. Zafar, M. Bakaul, and R. Parthiban, "Laser-Diode-Based Visible Light Communication: Toward Gigabit Class Communication," IEEE Commun. Mag., 55(2), 144-151 (2017).

[53]T. C. Wu, Y. C. Chi, H. Y. Wang et al., "Blue Laser Diode Enables Underwater Communication at 12.4 Gbps," Sci. Rep., 7, (2017).

[54]C. M. Lee, C. Zhang, M. Cantore et al., "4 Gbps direct modulation of $450 \mathrm{~nm} \mathrm{GaN} \mathrm{laser} \mathrm{for} \mathrm{high-speed} \mathrm{visible}$ light communication,” Opt. Express, 23(12), 16232-16237 (2015).

[55] C. Lee, C. Shen, H. M. Oubei et al., "2 Gbit/s data transmission from an unfiltered laser-based phosphor-converted white lighting communication system," Opt. Express, 23(23), 29779-29787 (2015).

[56] C. Lee, C. Zhang, D. L. Becerra et al., "Dynamic characteristics of $410 \mathrm{~nm}$ semipolar (20(2)over-bar(1)over-bar) III-nitride laser diodes with a modulation bandwidth of over 5 GHz," Appl. Phys. Lett., 109(10), 27-31 (2016).

[57] T. H. Matthew, M. K. Kathryn, L. You-Da et al., "m -Plane GaN-Based Blue Superluminescent Diodes Fabricated Using Selective Chemical Wet Etching,” Appl. Phys. Express, 2(12), 121004 (2009). 
[58] A. Kafar, S. Stańczyk, P. Wiśniewski et al., "Design and optimization of InGaN superluminescent diodes," Phys. Status Solidi (a), 212(5), 997-1004 (2015).

[59]C. Shen, T. K. Ng, C. Lee et al., "Semipolar InGaN-based superluminescent diodes for solid-state lighting and visible light communications," Proc. SPIE, 101041U-101041U-10 (2017).

[60]K. Fabian, E. Christoph, L. Alfred et al., "Blue Superluminescent Light-Emitting Diodes with Output Power above $100 \mathrm{~mW}$ for Picoprojection,” Jpn. J. Appl. Phys, 52(8S), 08JH07 (2013).

[61] A. A. Alatawi, J. A. Holguin-Lerma, C. H. Kang et al., "High-power blue superluminescent diode for high CRI lighting and high-speed visible light communication," Opt. Express, 26(20), 26355-26364 (2018).

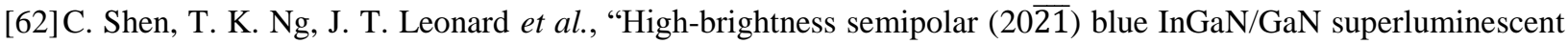
diodes for droop-free solid-state lighting and visible-light communications," Opt. Lett., 41(11), 2608-11 (2016).

[63]C. Shen, C. Lee, T. K. Ng et al., "High-speed 405-nm superluminescent diode (SLD) with 807-MHz modulation bandwidth," Opt. Express, 24(18), 20281-20286 (2016).

[64] J. A. Tatum, "Evolution of VCSELs," Proc. SPIE, 9001, (2014).

[65] M. Kuramoto, S. Kobayashi, T. Akagi et al., "High-output-power and high-temperature operation of blue GaNbased vertical-cavity surface-emitting laser," Appl. Phys. Express, 11(11), (2018).

[66] H. C. Yu, Z. W. Zheng, Y. Mei et al., "Progress and prospects of GaN-based VCSEL from near UV to green emission," Prog. Quant. Electron., 57, 1-19 (2018).

[67] C. Shen, J. T. Leonard, E. C. Young et al., "GHz modulation bandwidth from single-longitudinal mode violetblue VCSEL using nonpolar InGaN/GaN QWs," OSA Technical Digest. STh1L.2 (2016).

[68] L. A. Coldren, S. W. Corzine, and M. L. Mashanovitch, [Diode Lasers and Photonic Integrated Circuits] Wiley, (2012).

[69]Z. Y. Jiang, M. R. M. Atalla, G. J. You et al., "Monolithic integration of nitride light emitting diodes and photodetectors for bi-directional optical communication," Opt. Letters, 39(19), 5657-5660 (2014).

[70] W. Cai, X. M. Gao, W. Yuan et al., "Integrated p-n junction InGaN/GaN multiple-quantum-well devices with diverse functionalities," Appl. Phys. Express, 9(5), (2016).

[71] M. Tchernycheva, A. Messanvi, A. D. Bugallo et al., "Integrated Photonic Platform Based on InGaN/GaN Nanowire Emitters and Detectors," Nano Letters, 14(6), 3515-3520 (2014).

[72]C. Shen, C. Lee, T. K. Ng et al., "Integrated photonic platform based on semipolar InGaN/GaN multiple section laser diodes." 2017 Conference on Lasers and Electro-Optics Pacific Rim (CLEO-PR) (2017).

[73]C. Shen, T. K. Ng, J. T. Leonard et al., "High-Modulation-Efficiency, Integrated Waveguide Modulator-Laser Diode at $448 \mathrm{~nm}, "$ ACS Photonics, 3(2), 262-268 (2016).

[74]C. Shen, C. Lee, T. K. Ng et al., "GHz modulation enabled using large extinction ratio waveguide-modulator integrated with $404 \mathrm{~nm}$ GaN laser diode." 2016 IEEE Photonics Conference (IPC), 813-814 (2016).

[75]C. Shen, J. Leonard, A. Pourhashemi et al., "Low Modulation Bias InGaN-based Integrated EA-Modulator-Laser on Semipolar GaN Substrate," 2015 Photonics Conference (IPC), (2015).

[76]C. Shen, C. Lee, T. K. Ng et al., "High gain semiconductor optical amplifier - Laser diode at visible wavelength." 2016 IEEE International Electron Devices Meeting (IEDM), 22.4.1-22.4.4, (2016).

[77] C. Shen, T. K. Ng, C. Lee et al., "Semipolar InGaN quantum-well laser diode with integrated amplifier for visible light communications," Opt. Express, 26(6), A219-A226 (2018).

[78]C. Shen, C. Lee, E. Stegenburgs et al., "Semipolar III-nitride quantum well waveguide photodetector integrated with laser diode for on-chip photonic system," Appl. Phys. Express, 10(4), 042201 (2017).

[79]C. Shen, "Visible Lasers and Emerging Color Converters for Lighting and Visible Light Communications," OSA Technical Digest. SW3C.2 (2017).

[80] W. L. Hou, “A simple underwater imaging model,” Opt. Letters, 34(17), 2688-2690 (2009).

[81]H. T. Eyyuboglu, and Y. Baykal, "Scintillation characteristics of cosh-Gaussian beams," Appl. Optics, 46(7), 1099-1106 (2007).

[82] M. V. Jamali, A. Mirani, A. Parsay et al., "Statistical Studies of Fading in Underwater Wireless Optical Channels in the Presence of Air Bubble, Temperature, and Salinity Random Variations," IEEE Trans. Commun., 66(10), 4706-4723 (2018).

[83]E. Zedini, H. M. Oubei, A. Kammoun et al., "A New Simple Model for Underwater Wireless Optical Channels in the Presence of Air Bubbles," Globecom 2017 (2017).

[84]H. M. Oubei, X. Sun, T. K. Ng et al., "Scintillations of RGB laser beams in weak temperature and salinity-induced oceanic turbulence." Fourth Underwater Communications and Networking Conference (UComms) (2018) . 
[85] W. Cox, and J. Muth, "Simulating channel losses in an underwater optical communication system," J. Opt. Soc. Am. A, 31(5), 920-934 (2014).

[86]C. Gabriel, M. A. Khalighi, S. Bourennane et al., "Monte-Carlo-Based Channel Characterization for Underwater Optical Communication Systems," J. Opt. Commun. Netw., 5(1), 1-12 (2013).

[87] A. Laux, R. Billmers, L. Mullen et al., "The a, b, cs of oceanographic lidar predictions: a significant step toward closing the loop between theory and experiment," J. Mod. Opt., 49(3-4), 439-451 (2002).

[88] I. E. Lee, Y. K. Guo, T. K. Ng et al., "Bandwidth Enhancement of Wireless Optical Communication Link Using a Near-Infrared Laser over Turbid Underwater Channel," 2017 Conference on Lasers and Electro-Optics Pacific $\operatorname{Rim}(2017)$.

[89]I. E. Lee, Y. J. Guo, T. K. Ng et al., "Near-Infrared Wireless Optical Communication with Particulates InSuspension over the Underwater Channel," 2017 Conference on Lasers and Electro-Optics (CLEO), (2017).

[90]H. M. Oubei, R. T. ElAfandy, K. Park et al., "Performance Evaluation of Underwater Wireless Optical Communications Links in the Presence of Different Air Bubble Populations," IEEE Photon. J., 9(2), 1-9 (2017).

[91]H. M. Oubei, E. Zedini, R. T. ElAfandy et al., "Simple statistical channel model for weak temperature-induced turbulence in underwater wireless optical communication systems," Opt. Letters, 42(13), 2455-2458 (2017).

[92]B. Cochenour, L. Mullen, and A. Laux, "Phase Coherent Digital Communications for Wireless Optical Links in Turbid Underwater Environments." OCEANS 2007 (2007).

[93] S. Tang, Y. Dong, and X. Zhang, "On path loss of NLOS underwater wireless optical communication links." 2013 MTS/IEEE OCEANS - Bergen (2013).

[94] W. Liu, D. Zou, Z. Xu et al., "Non-line-of-sight scattering channel modeling for underwater optical wireless communication." 2015 IEEE International Conference on Cyber Technology in Automation, Control, and Intelligent Systems (CYBER), 1265-1268 (2015).

[95]C. Gabriel, M. Khalighi, S. Bourennane et al., "Monte-Carlo-based channel characterization for underwater optical communication systems," J. Opt. Commun. Netw., 5(1), 1-12 (2013).

[96]C. D. Mobley, [Light and water: radiative transfer in natural waters] Academic press, (1994). 